Journal of Engineering and Applied Sciences 14 (13): 4375-4386, 2019

ISSN: $1816-949 \mathrm{X}$

(C) Medwell Journals, 2019

\title{
Dynamic Routing Algorithm for Spatio-Temporal Query Processing in Sensor Networks
}

\author{
Jeong-Joon Kim \\ Department of Computer Science and Engineering, Korea Polytechnic University, \\ Gyeonggi-do, 15073 Siheung-si, South Korea
}

\begin{abstract}
A GR-tree method and a query aggregation(Query Aggregation) method have been proposed for spatial query processing with space query processing techniques for existing wireless sensor networks. However, such a spatial query processing technique considered the optimization of spatial queries but it was not taken into consideration about the question and answer of time and due to the increase of the index reorganization cost and the communication cost of the parent sensor node. There is a problem that the energy consumption that requires the most efficiency increases at the wireless sensor node. In this study, we solve the problem of such a spatial query processing technique and investigate the IR-tree (Itinerary based R-tree) for the more efficient time space (Spatiotemporal) Present.
\end{abstract}

Key words: Sensor networks, dynamic routing algorithm, spatio-temporal, R-tree, MBR, optimization, itinerary based R-tree

\section{INTRODUCTION}

The wireless sensor network is composed of a plurality of sensor nodes (sensor nodes). Sensor nodes are composed of sensing, processing, storage and communicating modules and the use of hardware and software resources is extremely limited. Especially, energy capacity is very small and energy consumption during data transfer is high. Therefore, related studies for efficiently processing spatio-temporal query in environments matching the characteristics of these sensor nodes are actively performed (Gang et al., 2007; Kim and Youn, 2010; Kim and Jang, 2011; Soo et al., 2007; Culler et al., 2004).

Spatio-temporal operation query processing is a method of processing queries such as temperature, humidity and illuminance for the entire wireless sensor network or a specific area. The simplest method of these sensor network based spatio-temporal query processing is the centralized method of collecting the location and sensing information of all sensor nodes to the server and processing the query of the space on the server. Although, this method has an advantage of being simple it has a disadvantage that the energy efficiency of the sensor node is greatly reduced due to the high wireless communication cost required for accessing all the sensor nodes. Therefore, in order to complement the centralized method, an in-network based distributed spatial index method has been proposed that performs distributed spatial filtering at the sensor node to reduce the number of wireless communications between sensor nodes. However, since, these distributed spatial index methods simply apply the existing spatial index method used by most servers to the wireless sensor network, the effects of spatial filtering and wireless routing between sensor nodes are simultaneously optimized in the in-network environment. There are no disadvantages and a GR-tree method complementing this has been presented (Kim and Jang, 2011).

In this study, we solve the problem of such a spatial query processing technique and present IR-tree (Itinerary based R-tree) for more effective query processing in wireless sensor network. In the creation of the IR-tree in the specific sensor space region, the center node (Center Node) is first, selected according to the sensor node distribution to form an R-tree based on MBR (Minimum Bounding Rectangle). The MBR of the parent node may overlap between the MBRs of the R tree and the MBR of the parent node is a hierarchical tree structure including the MBR of the child node. Index construction the upper certain level during query processing performs an R-tree search and uses sub-mostly Itinerary method (Guttman, 1984; Akkaya and Younis, 2005). Also, we derive optimal R-tree index level through self performance evaluation at the time of data acquisition.

\section{Literature review}

IWQE (Itinerary based Window Query Execution): IWQE is a space arithmetic query processing technique in the network using itinerary routing for spatial operation query processing (Kim et al., 2011). IWQE does not set the routing of the whole area of the wireless sensor network 


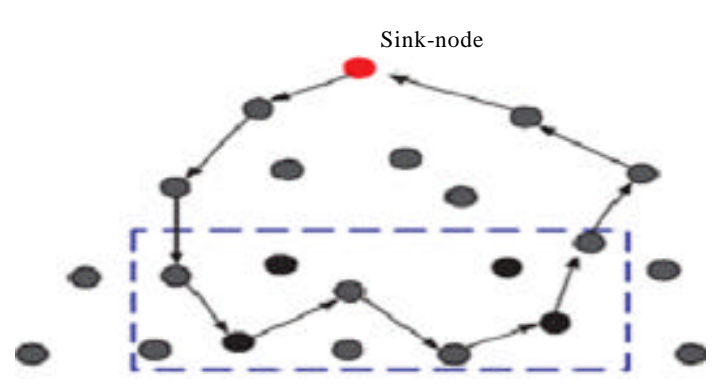

Fig. 1: Itinerary routing structure of IWQE

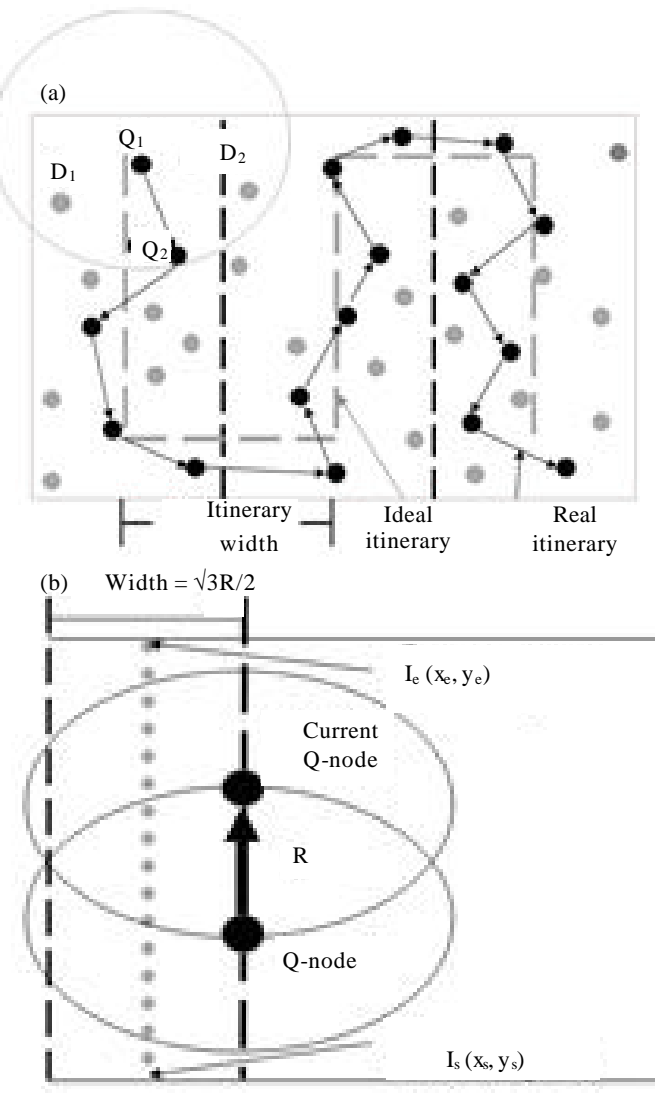

Fig. 2: a, b) Ideal itineraries and real itineraries of IWQE

in advance, temporarily configures the routing only in the quality range (Query Range) and processes the query at the time when the user's query is given. Figure 1 shows an itinerary routing structure of IWQE.

As shown in Fig 1, IWQE uses itinerary routing to query the sensing data of the sensor nodes in the quality domain and the sink node returns the result of the final query to the server Fig. 2 shows an itinerary routing example of IWQE.

Within the quality domain in Fig. 2, the sensor node has one of the characteristics of the Q-node transmitting the query and the D-node transferring the data of the

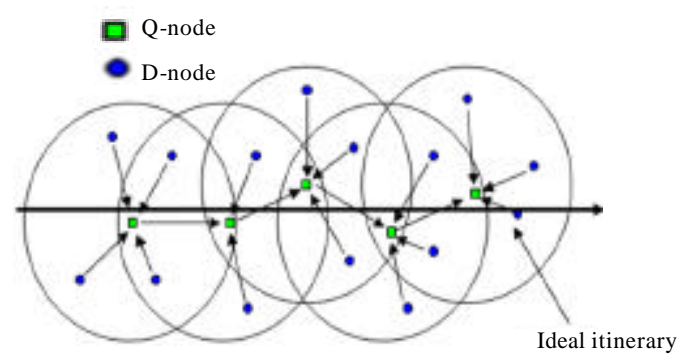

Fig. 3: IWQE aggregate operation query processing example

query result and executes the query based on the ideal itinerary. At this time, the actual routing path is set to real itinerary and the itinerary routing interval width is set using the communication range $\mathrm{R}$ of the sensor node. Itinerary width means the distance between ideal itineraries and means the distance between real itineraries.

Based on itinerary routing within the quality domain at IWQE, the Q-node sends a query to the $\mathrm{D}$ node and the D-node sends the result of the query to the Qnode Fig. 3 shows an example of query processing of IWQE.

From Fig 3, the Q-node queries the D-node in the communication range and the $\mathrm{D}$-node sends the query result to the Q-node. The Q-node then sends the result of the intermediate summary query to the next Q-node. At this time, based on the ideal itinerary of the sensor node within the communication range of the Q-node, a sensor node that can maximize progress is selected as the next Q-node. In addition, the Q-node transfers data to the next Q-node by a unicast method in order to reduce loss of data due to transfer errors at the time of data transfer and the Dnode transmits data to the Q-node transfer the data by the broadcast method.

IWQE does not have unnecessary energy consumption of sensor nodes that are not included in the quality area and there is no routing maintenance fee. And if the quality area is small or there are few sensor nodes it is efficient. However, since, duplicate data is generated by broadcast transmission to reduce data transfer error, the accuracy of the query processing result drops and the energy consumption of the sensor node is large. When there are many sensor nodes and the quality area is large there is a problem that it takes time for query processing.

R-tree: In this study, we consider an R-tree that extends the B-tree multidimensionally to efficiently store and manage various multidimensional spatial data (Guttman, 1984; Akkaya and Younis, 2005; Bash et al., 2004). Nodes 

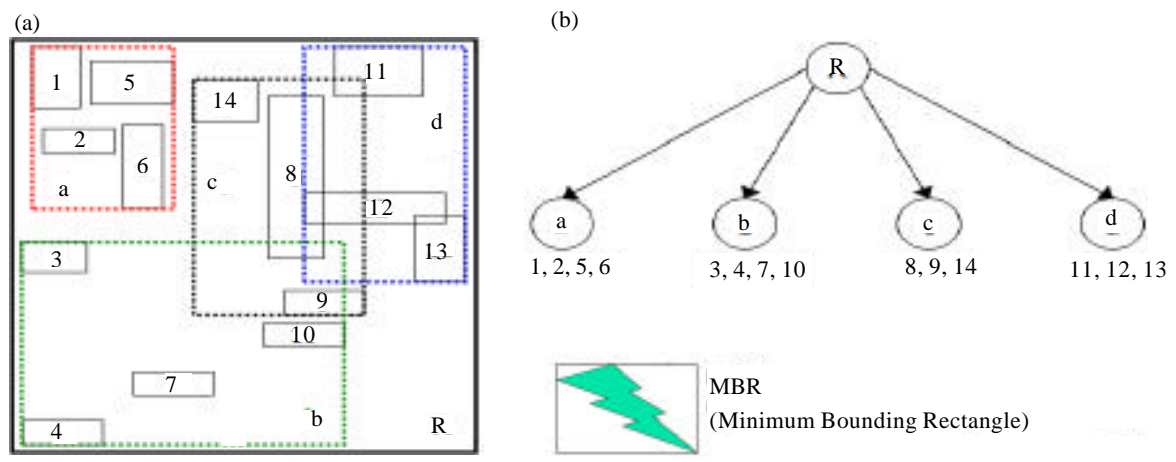

Fig. 4: a, b) Example of R-tree structure

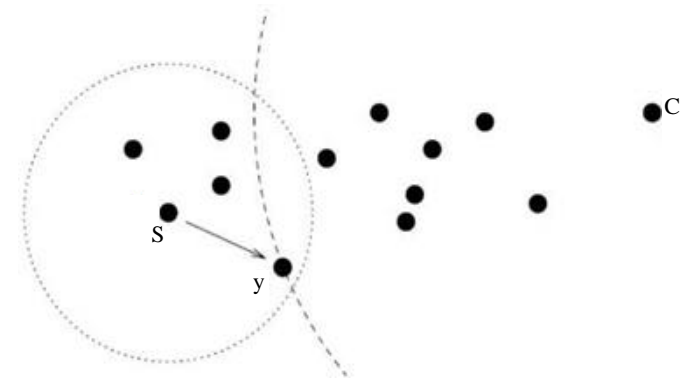

Fig. 5: Example of greedy forwarding

other than the root node of the R-tree include maximum $\mathrm{M}$, Minimum $m(m \leq M / 2)$ index entries and child pointers. $M$ is the Maximum number of entries a node can hold and $\mathrm{m}$ is the minimum number of entries a node can hold. Since, the root node is not a leaf it has at least two child nodes. The entry of the intermediate node consists of a larger MBR containing all the MBR's of the child node and a pointer to the child node. Each index entry of the leaf node includes the address of the page in which the spatial object is stored and the MBR in which the data of the object is actually spatially included. R tree As shown in Fig. 4 it is a fully balanced tree structure.

GPSR (Greedy Perimeter Stateless Routing): Since, the radio range is limited, communication between a sensor node of a source on a network composed of the entire wireless sensor node and a sensor node of a destination requires passing through a plurality of nodes. The GPSR algorithm is an algorithm that selects the node closest to itself. The departure sensor node repeats the algorithm of selecting the position sensor node nearest to itself at an intermediate stage of knowing the address of the destination node.

In order to select a path from Fig. 5, the GPSR uses the greedy forwarding algorithm. The wireless sensor node knows the position of the node in the vicinity of the

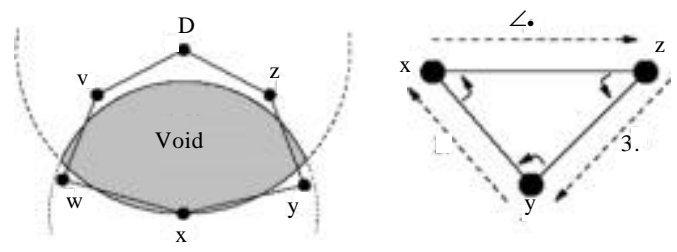

Fig. 6: Right hand rule

communication radius with itself and recognizes the destination node using the greedy forwarding algorithm. Figure 5 shows a greedy forwarding example which is the process of searching the route at the shortest distance to the C-node at the S-node.

In the GPRS algorithm if there are two paths ( $\mathrm{x}-\mathrm{w}-\mathrm{v}-\mathrm{D}$, $\mathrm{x}-\mathrm{y}-\mathrm{z}-\mathrm{D})$ from the $\mathrm{X}$-node to the $\mathrm{D}$-node as shown in Fig. 6 and they are the same, the right hand rule is applied and the $x-y-z-D$ path is searched. Figure 6 shows an example of $\mathrm{y}-\mathrm{x}-\mathrm{z}$ right hand rule radio waves (Karp and Kung, 2000).

\section{MATERIALS AND METHODS}

\section{Algorithm dsign}

Hypothesis and terms: This study explains the assumptions assumed during spatio-temporal query processing and the terms defined for IR-tree design. In this study, we assume the following five assumptions at the time/space quality processing.

- The wireless sensor node is not moving but is fixed and sensor nodes can be added and deleted

- All sensor nodes have the same communication range and can mutually communicate within the communication range

- The sensor node knows its own geometric position by utilizing adjacent nodes in the communication range, RFID and GPS 
- The sensor node knows at least the current transfer sensing data and the sensing data sent just before

- The area of the sensor network where all sensor nodes are located is fixed

Table 1 is defined in this study for IR-tree design and shows terminology.

Create index: For spatio-temporal query processing, the IR-tree first collects information on sensor nodes constituting R-trees of all wireless sensor nodes in the sensor area. Because of the R-tree configuration, the sensor node closest to the center point of the sensor network area (SR) is searched and selected by the routing root node and this node becomes the center node. Since, the area of the sensor network is known in advance, the center point can be obtained by calculation. Figure 7 shows the process of selecting the Central node (C-node) which is the root sensor node of the $\mathrm{R}$-tree routing.

Figure 7 shows the $\mathrm{C}$-node selection process starting from the S-node (Base station) which is the starting sensor node of quality. Each sensor node on the route calculates the distance between the center point of the area of the sensor network and its own sensor node and calculates the distance between the center point of the sensor network area and the neighbor sensor node within its communication range. If the sensor node is more centrally located than its own sensor node, the query is forwarded to the adjacent sensor node and when the sensor node is closer to the center than the adjacent sensor node it is selected and returned to the $\mathrm{C}$-node.

The spatio-temporal quality data structure consists of a quality id an area representing the coordinates of the query area qt indicating the time of the question and question, qa representing the question and answer attribute, agg indicating the type of the aggregation operation, frequency indicating the query processing result transmission cycle, sensor node S-node (id, pos) indicating the information of the node. The R-tree and itinerary warning level information propagate information to all sensor nodes at the first time after configuring the first R-tree and all the sensor nodes save information of the sensor node or R-tree routing information data reflect on the structure. The IR-tree constructs R-tree routing for all the wireless sensor nodes in the area of the sensor network as a whole with the selected C-node as the root and selects the optimal level boundary between the R-tree and itinerary Fig. 8 shows an R-tree routing structure.

As shown in Fig. 8, the route and silk word sensor node has a plurality of child sensor nodes and the child
Table 1: Term

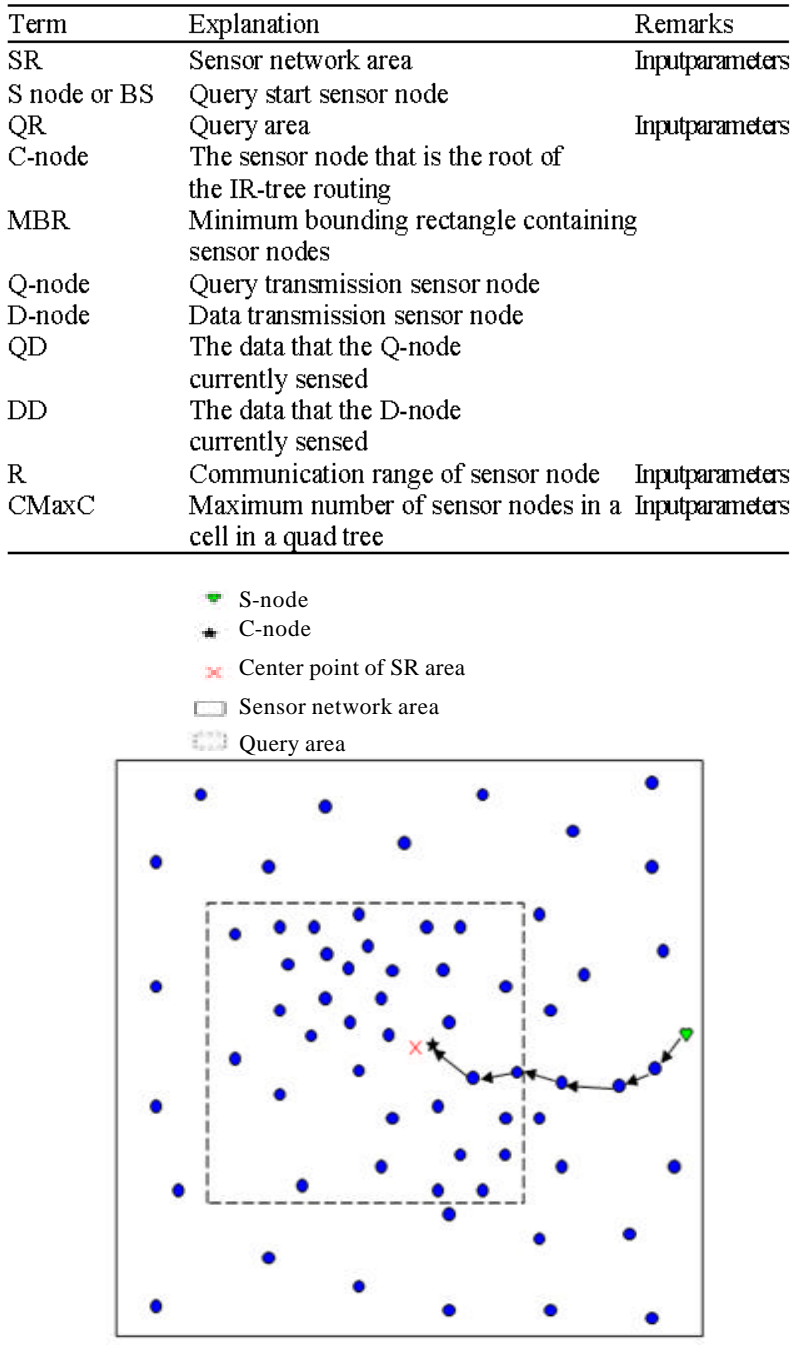

Fig. 7: C-node selection process

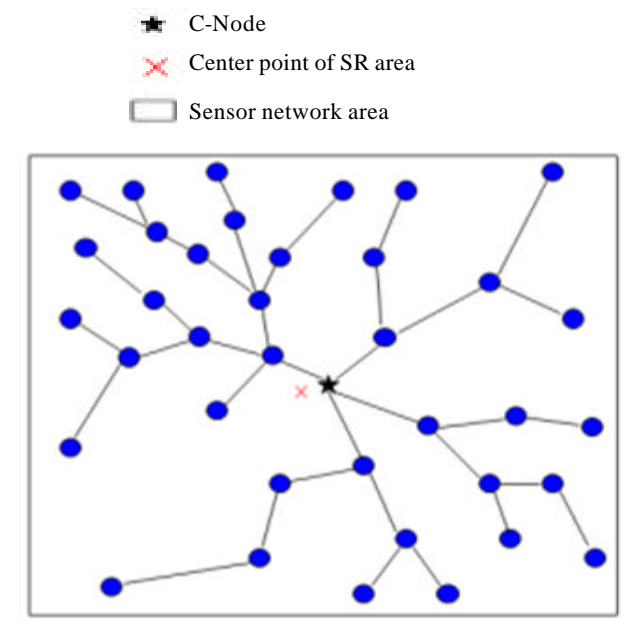

Fig. 8: R-tree routing structure 
sensor node has only one parent sensor node. Then, the child sensor node selects the sensor node closest to the root node as the parent sensor node. If there are a plurality of sensor nodes closest to the route sensor node, the sensor node having the largest amount of remaining energy is selected as the parent sensor node.

In order to select the parent node, as shown in Fig. 9, broadcast BM (Build Message) to all nodes within the communication radius from the root node. The node that has received the BM rebroadcasts the $\mathrm{BM}$ to all nodes within its communication radius, except for the node that sent the BM. Continue execution to the terminal node. The BM message includes the transmission node $\mathrm{id}$, the position of the transmission node, the position of the BS and the depth information of the transmission node and the BS.

Configure an R-tree sensor node (MBR Minimum Boundary Rectangle) and collect information on sensor nodes. Figure 9 shows a configuration example of MBR between sensor nodes.

Starting from the C-node which is the route sensor node of the R-tree routing as shown in Fig. 10, the sensor node having the child node constitutes the minimum circumscribing square MBR including the sensor node of its child. Then, information on the sensor node in the MBR area is collected and transmitted to the parent sensor node Fig. 11 shows an example of the data structure and information of the sensor node.

As shown in Fig. 11, the data structure of the sensor node information includes SInfo indicating its own sensor node information (ID, POS), MBR representing the minimum circumscribing rectangle including its own and child sensor nodes, count indicating the number of sensor nodes and pointer* Clist [] pointing to child sensor node information. The lower left point of the MBR is set using the smallest $\mathrm{x}, \mathrm{y}$ coordinates of the position information of the sensor node of itself and the child and the idle point is set within the position information of the sensor node of itself and the child. It is set using the largest $\mathrm{x}$ and $\mathrm{y}$ coordinates. After configuring $\mathrm{MBR}$, propagate $\mathrm{R}$-tree and itinerary routing level boundary value to all nodes.

The IR-tree reconstructs the entire R-tree when the area of the sensor network is changed. However, since, IR-tree consists of itinerary method under specified specific index level when adding and deleting sensor nodes, updating is unnecessary and on top of certain levels periodic Reconstruction of the R-tree is necessary. Although, it has the disadvantage that cost of reconstruction of $\mathrm{R}$-tree accompanying MBR updating is required it is expected to cost less than the conventional method in terms of overall efficiency and it is demonstrated by its own performance evaluation. The insertion and deletion algorithms apply the conventional R-tree update algorithm.

Spatio-temporal merging: Next, we show an example of merging space (region), attribute and time for spatiotemporal queries and the Question $Q$ is composed of $<$ region, attribute, time etc.

Figure 12 is before the merge of contains. A later example will be explained. That is we show an example of merge of space, attribute and time for two queries. $Q_{1}$ send a $Q_{1}$ Query in a relationship where the Query contains $Q_{2}$ quality completely:

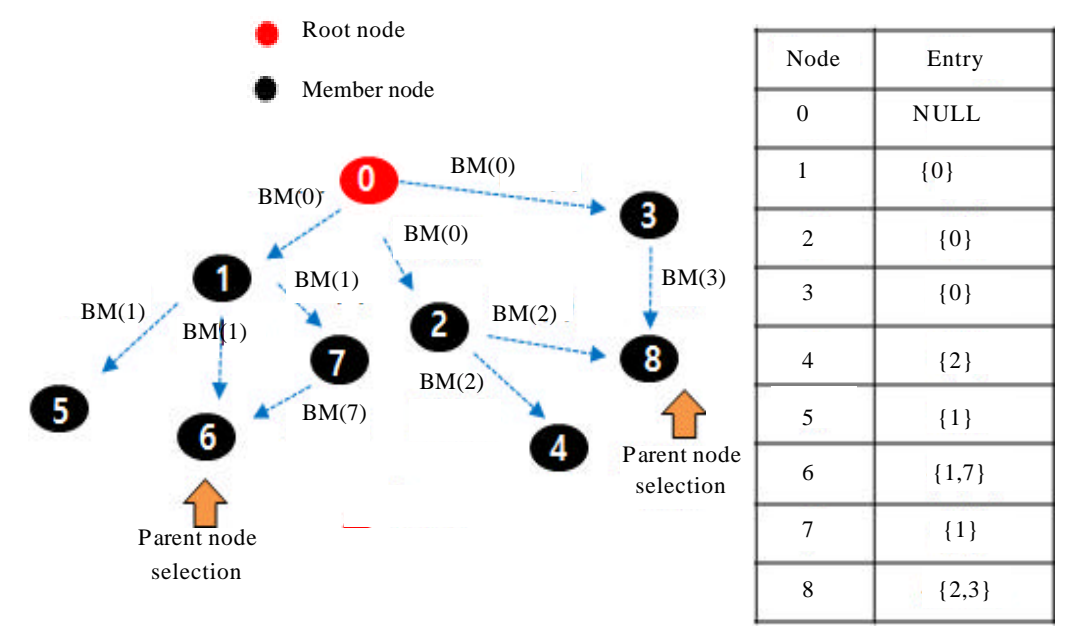

Fig. 9: Send BM message 


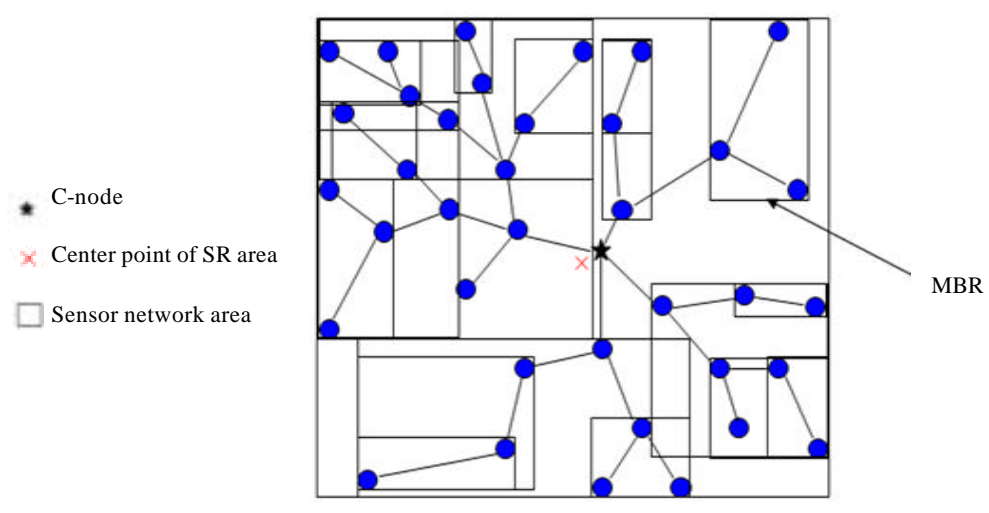

Fig. 10: Configuration example of MBR between sensor nodes

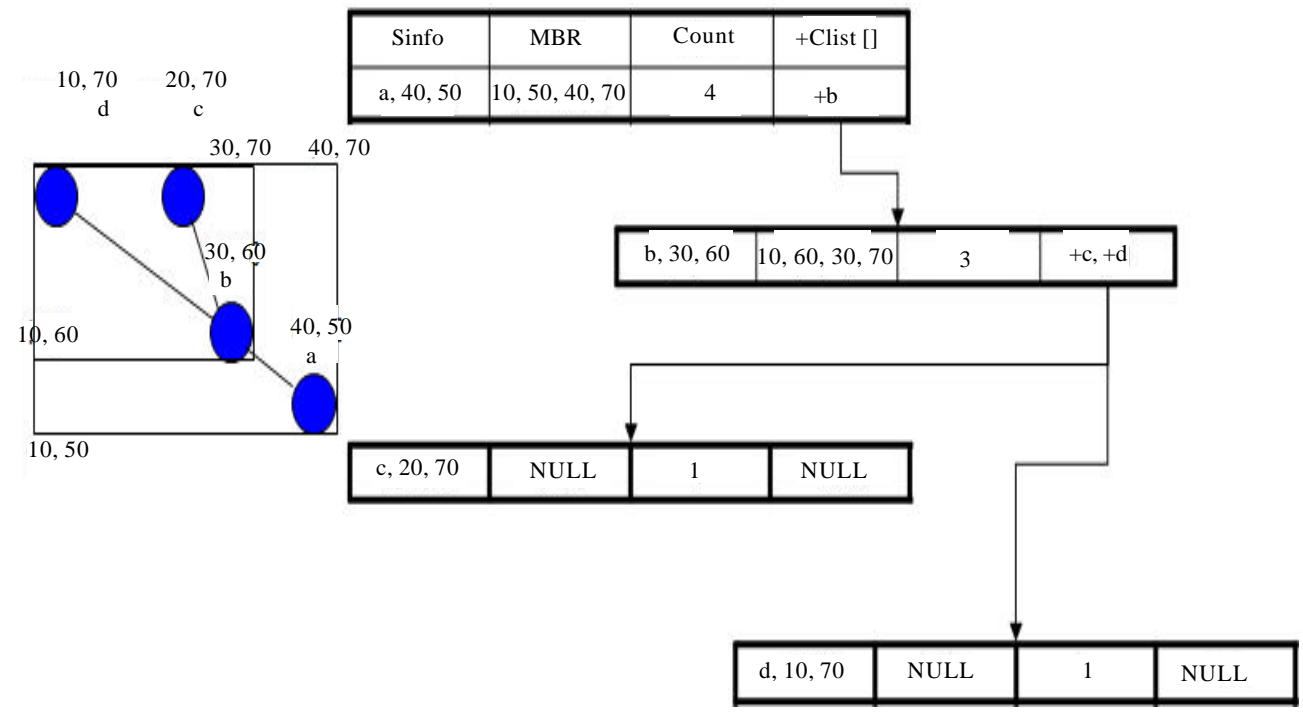

Fig. 11: Sensor node information data structure and example

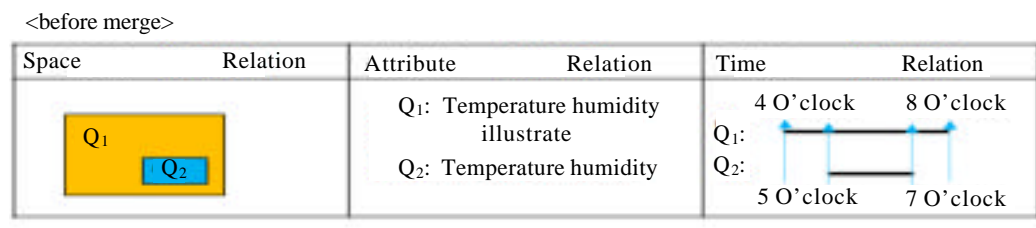

\begin{tabular}{|c|c|c|c|c|}
\hline Space & Relation & Attribute & Time & Relation \\
\hline Q & & $\begin{array}{l}\text { Q1: Temperature humidity } \\
\text { illustrate }\end{array}$ & \multicolumn{2}{|c|}{$\begin{array}{l}4 \text { O'clock } 8 \text { O'clock } \\
\mathrm{Q}_{1}:{ }^{-}\end{array}$} \\
\hline \multicolumn{2}{|c|}{$\begin{array}{l}\mathrm{Q}_{1}: \text { Space contains } \\
\mathrm{Q}_{2}: \text { Space }\end{array}$} & $\begin{array}{l}\mathrm{Q}_{1}: \text { Attribute contains } \\
\mathrm{Q}_{2}: \text { Attribute }\end{array}$ & \multicolumn{2}{|c|}{$\begin{array}{l}\mathrm{Q}_{1} \text { : Time contains } \\
\mathrm{Q}_{2} \text { : Time }\end{array}$} \\
\hline \multicolumn{5}{|c|}{$\begin{array}{l}\text { Report the temperature humidity and illuminance from } 4-8 \text { O' clock every } 5 \mathrm{~b} \text { sec in the area } \\
\{(10,10),(50,50)\}\end{array}$} \\
\hline
\end{tabular}

Fig. 12: Contains merge example 


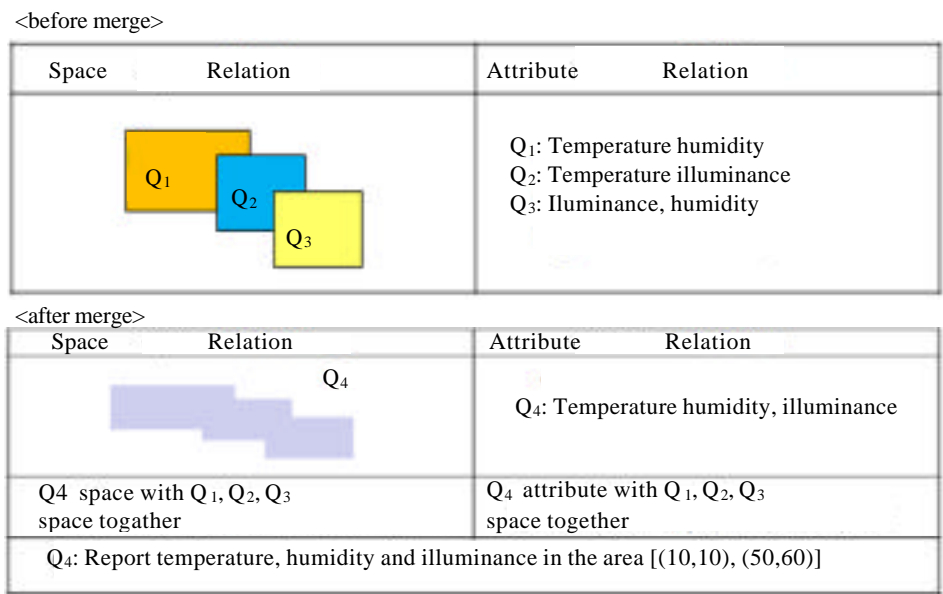

Fig. 13: Overlap spatial merge example

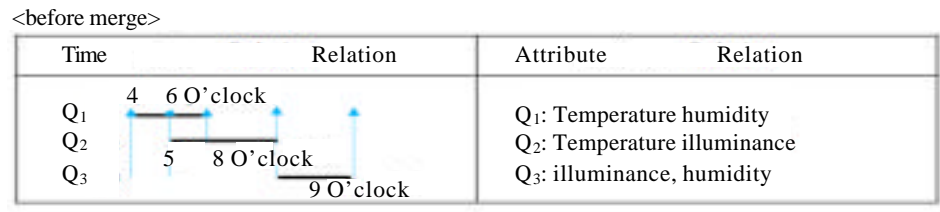

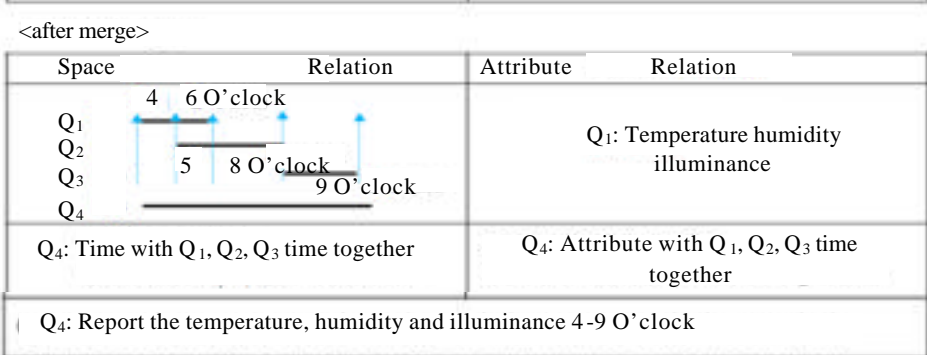

Fig. 14: Overlap time merge example

- $\mathrm{Q}_{1}:$ Report the temperature, humidity, illuminance from $4-8$ O'clock every $5 \mathrm{sec}$ in the area $[(10,10),(50,50)]$

- $\mathrm{Q}_{2}$ : Report the temperature and humidity from 5-7 O'clock every $10 \mathrm{sec}$ in the area $[(20,20),(40$, 30)]

Figure 13 is before the merge of overlap space. A later example will be explained. That is we show an example of merging space and attributes for three queries. Together with the space including both the space of $Q_{1}-Q_{3}$ and the attribute, the new Query $\mathrm{Q}_{4}$ is transmitted:

- $\mathrm{Q}_{1}$ : Report the temperature and humidity in the area $[(10,10),(30,40)]$

- $\mathrm{Q}_{2}:$ Report the temperature and illuminance in the area $[(20,30),(40,50)]$

- $\mathrm{Q}_{3}$ : Report illuminance and humidity in the area $[(35$, $40),(50,60)]$

Figure 14 is before the merge of overlap time. A later example will be explained. In other words, we show examples of time and attribute merging for three queries. And transmits a new query $Q_{4}$ including both time and attribute including both $\mathrm{Q}_{1}-\mathrm{Q}_{3}$ time:

- $\mathrm{Q}_{1}$ : Report temperature and humidity from 4-6 O'clock

- $\mathrm{Q}_{2}$ : Report temperature and illuminance from 5-8 O'clock

- $\mathrm{Q}_{3}$ : Report illuminance and humidity from 8-9 O'clock

\section{RESULTS AND DISCUSSION}

In this study, we describe our own performance evaluation. In the original performance evaluation when the spatio-temporal coupling is allowed, the accuracy of the sensor node query processing result of the IR-tree corresponding to the itinerary configuration level, the energy consumption of the wireless sensor node, the performance of the query processing time of the wireless sensor node are evaluated. In the case of uniform 

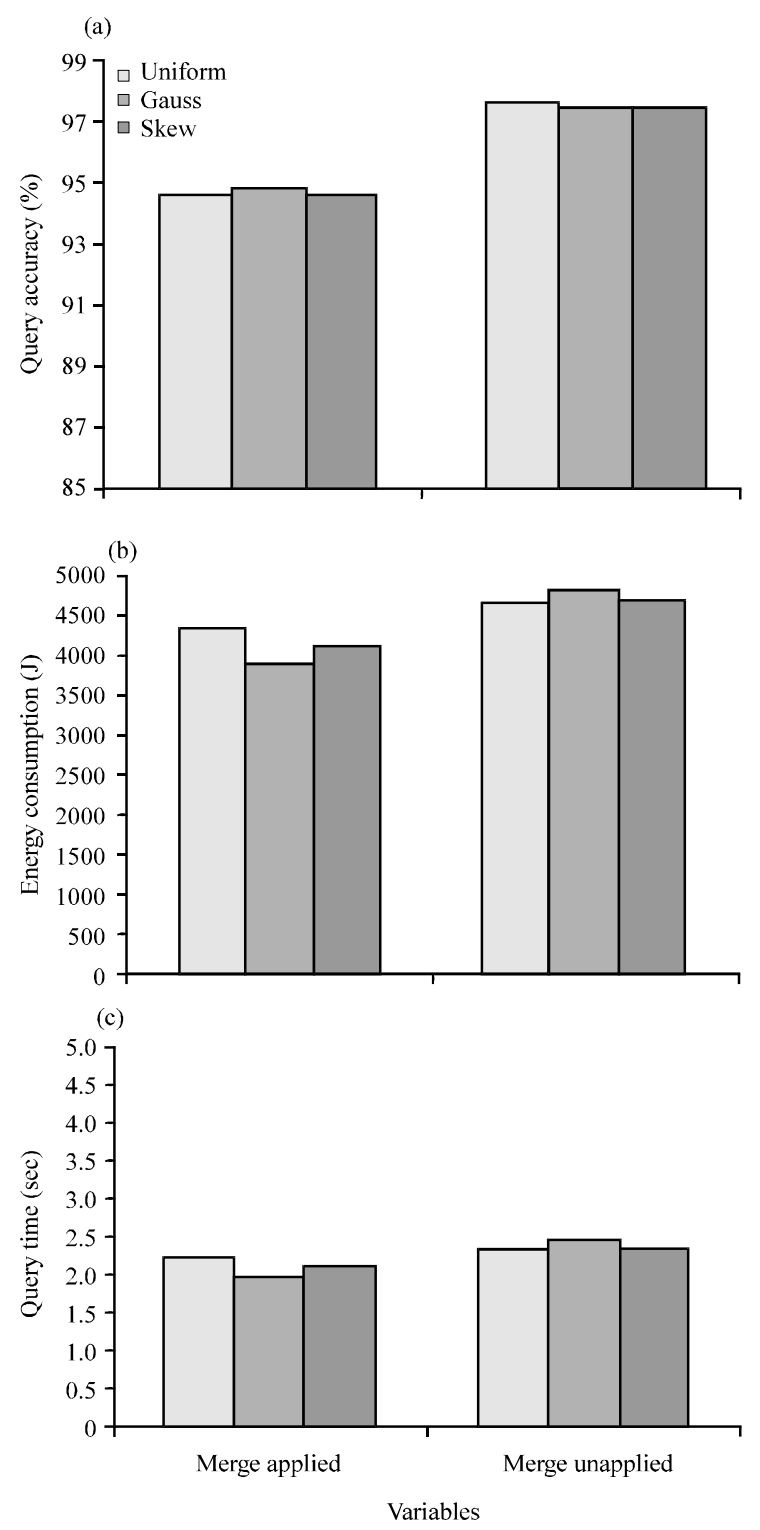

Fig. 15: a-c) Performance evaluation according to spatio temporal merging

distribution, IR-tree and pure R-tree evaluate the accuracy of pure itinerary interstitial processing result, energy consumption and query processing time.

Performance evaluation according to spatio-temporal merging: In the performance evaluation accompanying permission of coupling of spatio-temporal quality we compare the case of applying spatio-temporal coupling during query execution with uniform distribution, Gauss distribution, Skew distribution and not applying spatio-temporal coupling. In the self performance evaluation, the number of continuous query questions of the sensor node is 600 times, the size of the query area is $990^{*} 990 \mathrm{~m}^{2}$ and the number of sensor nodes is 60 thou. pieces.

Figure 15 shows the results of the performance evaluation accompanying permitting spatio-temporal coupling.

As shown in Fig. 15a, the accuracy of the query processing result is somewhat inferior to the case where it is not applied when spatio-temporal coupling is applied but shows the result of the performance evaluation of $95 \%$ or more as a whole it was. As shown in Fig. 15b, the energy consumption of the sensor node is $7 \%$ on average in the case of uniform than in the case where it is not applied when the spatio-temporal coupling is applied, 24\% in the case of Gauss for Skew, the average 14\% performance has been improved. Then as shown in Fig. 15c, the query processing time is $4 \%$ on average in the case of uniform than in the case of not applying when the coupling of spatiotemporal quality is applied, $22 \%$ in the case of Gauss, $22 \%$ in the case of Skew an average of $10 \%$ performance was improved.

The results of these performance evaluations show that when spatio-temporal coupling is applied, instead of collecting sensor node data to their respective qualities we can perform spatio-temporal quality combining and calculate the number of queries. This is because the sensor node's data transfer count has decreased. Therefore, when applying spatio-temporal binding, the accuracy of the results of queries is somewhat lower than when not applying spatio-temporal question merging but the overall energy consumption which is very important in application to sensor nodes is small, processing time is small. And these performance differences were evaluated to be even larger, if the sensor nodes were Gauss, Skew distribution.

Performance evaluation according to itinerary configuration level: In the performance evaluation according to the itinerary configuration level when constructing the IR-tree with the uniform distribution, Gauss distribution, Skew distribution, set the Itinerary configuration level to $0,30,40,50,60,70,75,80,85,90$, 95 and $100 \%$. Especially, there are many changes, over $70 \%$, the interval between the sections was $5 \%$. Figure 16 shows the results of the performance evaluation according to the Itinerary configuration level.

As shown in Fig. 16a, the precision of the query processing result shows that the Itinerary configuration level shows accuracy of $95 \%$ or more up to about $70 \%$ of the height of the entire index and decreases from $70 \%$ onward to below $95 \%$. The $0 \%$ level was more accurate than the $100 \%$ level. As shown in Fig. $16 \mathrm{~b}$ and $\mathrm{c}$ the 


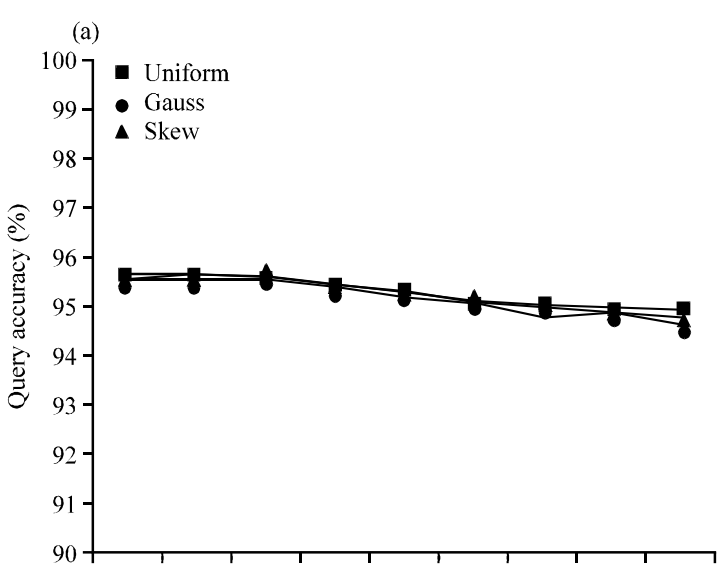

(b)

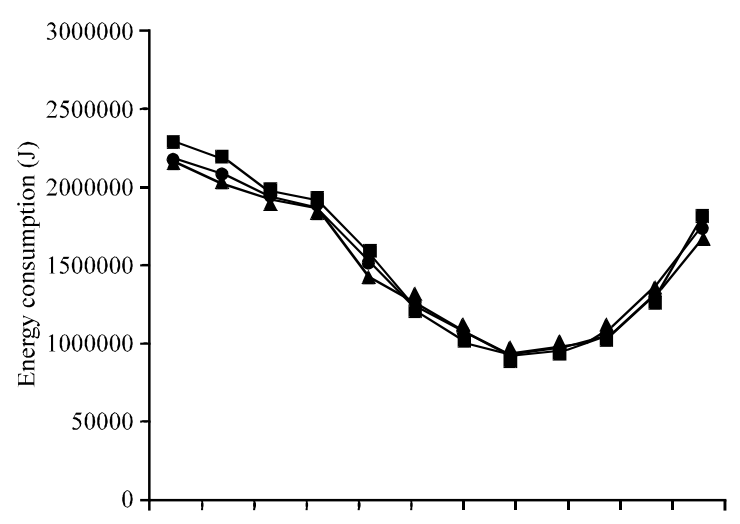

(c)

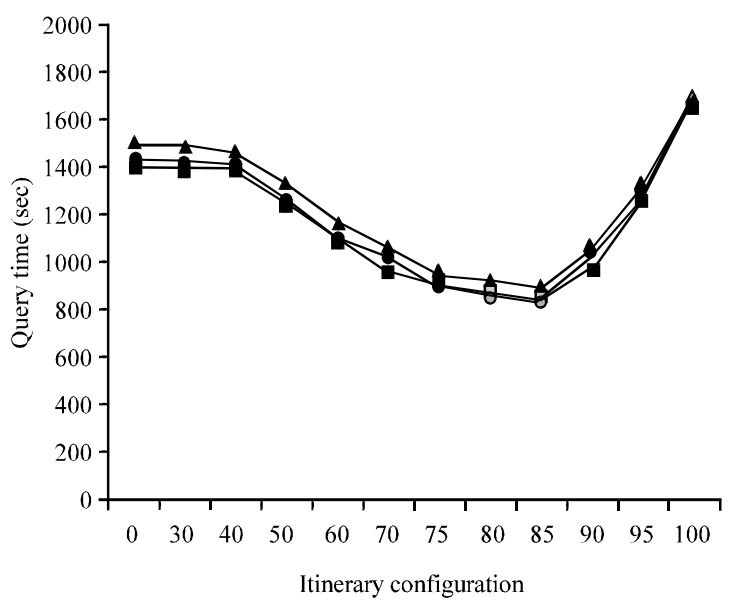

Fig. 16: a-c) Performance evaluation according to itinerary configuration level

higher the itinerary configuration level is the lower the energy consumption, the query processing time shows the high speed performance evaluation result but it exceeds the level of $80 \%$, again the energy consumption increased and the query processing time was delayed again after exceeding the $85 \%$ level. Itinerary from R-tree. It consumes less energy, itinerary than R-tree it took time to process.

As a result of these performance evaluations, the higher the itinerary configuration level is the lower the precision of query processing is. However, since, the index restructuring cost which is the merit of itinerary and the energy consumption of a specific parent node are small, the energy of the node of the entire sensor even if consumption is reduced, the processing time for parallel processing is reduced in the Itinerary area. However, it was found that the number of nodes in the itinerary is larger in the case of a specific configuration level or more, energy consumption and processing time increase, eventually increasing energy consumption and processing time of the IR-tree. However, since, the reliability of the query processing result is not guaranteed when the accuracy of the query processing drops too much, it is necessary to specify the proper level of itinerary. In the performance evaluation of the book itself while maintaining the accuracy of $95 \%$ or more when the itinerary configuration level is about $70 \%$ of the height of the overall index, the processing time of energy consumption and quality also shows a somewhat good performance evaluation result it was.

Performance evaluation according to itinerary configuration level: In this study, IR-tree and pure R-tree for the case of uniform distribution performance evaluate energy consumption between pure itinerary, accuracy of query processing result and query processing time. Pure Rtree and pure itinerary are not all quality coupled. Do not process queries in parallel in the quality domain, sequentially. In this evaluation, the performance is best when the $\mathbb{R}$-tree itinerary level is $80-85 \%$ but the itinerary level is set to $70 \%$ in order to maintain the accuracy of $95 \%$ or more. With the performance evaluation according to the number of sensor nodes, the number of continuous questioning is 600 times and the size of the query area is $990^{*} 990 \mathrm{~m}^{2}$ while increasing the number of sensor nodes from $20,000-100,000$ by 20,000 at a time, performance evaluation was carried out.

Figure 17 shows the energy consumption according to the number of nodes between IR-tree, Rtree and itinerary, the accuracy of the query processing result and the performance evaluation of the query processing time.

In the performance evaluation according to the number of consecutive questions, the number of sensor nodes is 60,000 the size of the query area is $990^{*} 990 \mathrm{~m}^{2}$ while the 

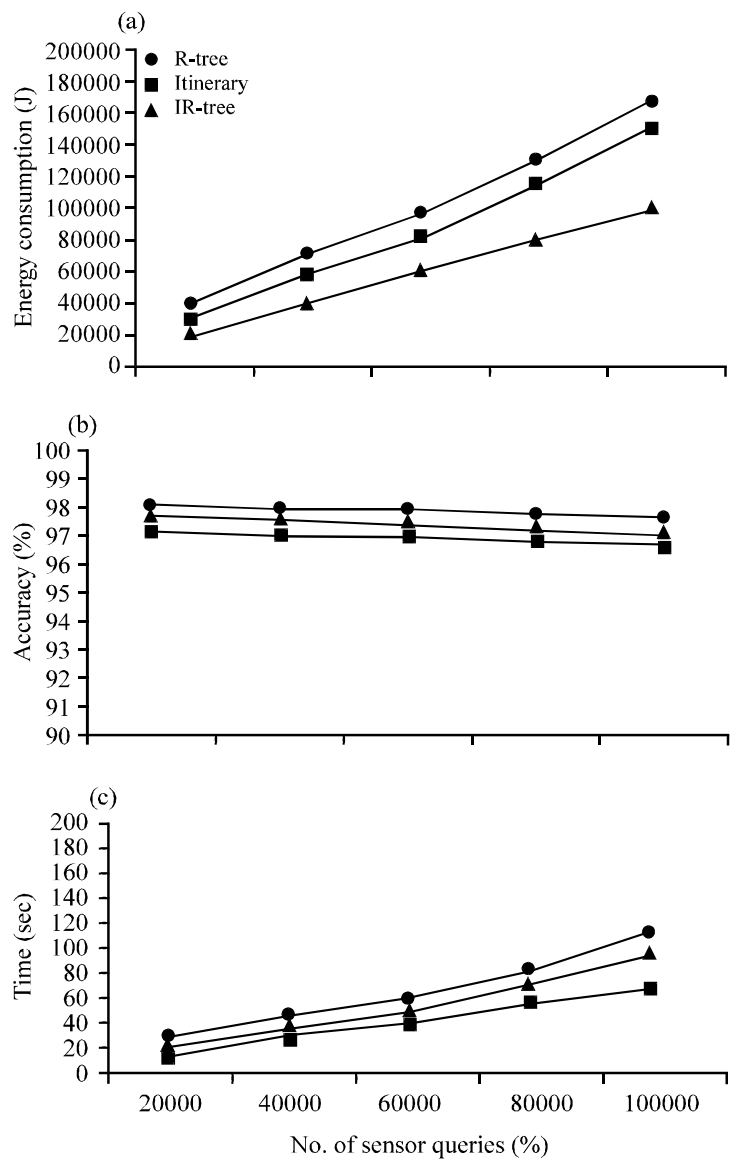

Fig. 17: Performance evaluation according to the number of sensor nodes: a) Energy consumption; b) Accuracy of processing results and c) Querry processing time

number of continuous questions is increased by $200-1000$ times by 200 times while performing performance evaluate. Figure 18 shows the energy consumption by IR-tree, Rtree, itinerary interstitial frequency, accuracy of query processing result and performance evaluation of query processing time.

In the performance evaluation corresponding to the size of the quality area, the number of sensor nodes is 60,000 the number of continuous questioning is 600 and the size of the question area is increased by $330 * 330 \mathrm{~m}^{2}$ by $330 * 330-$ $1,650^{*} 1,650 \mathrm{~m}^{2}$ performance evaluation was carried out Fig. 19 shows the energy consumption according to the size of the region between the IR-tree, the R-tree and the itinerary, the accuracy of the query processing result and the performance evaluation of the query processing time.

As shown in Fig. 17-19, the IR-tree has the least energy consumption, the accuracy is highest in the R-tree and the query processing time shows the result of the performance evaluation with the $\mathbb{R}$-tree at the highest
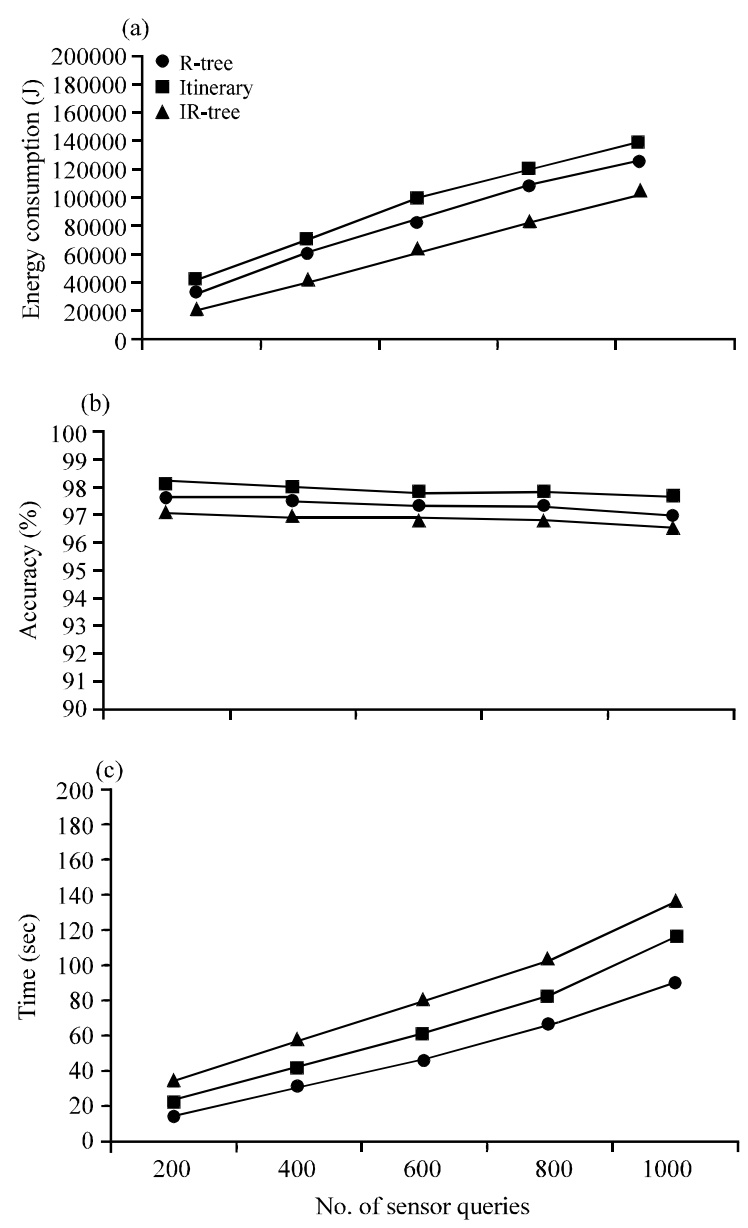

Fig. 18: Performance evaluation according to the number of continuous sensor query: a) Energy consumption; b) Accuracy of processing results and c) Querry processing time

speed it was. And itinerary was somewhat slower in query processing time than R-tree energy consumption was shown in Fig. 20 as a result of less performance evaluation.

As a result of these performance evaluations, since, the R-tree is a static hierarchical routing structure, although, the accuracy of query processing is high, energy consumption is high and the processing time is an intermediate position. Since, itinerary is a dynamic routing structure, query processing time is slow and query processing accuracy is low but energy consumption is an intermediate position.

The proposed IR-tree proposed in this study accepts the advantages of these hierarchical routing and dynamic routing structures while minimizing disadvantages, so, the accuracy of query processing somewhat falls somewhat but the energy consumption and quality, the evaluation results of excellent performance were shown. 

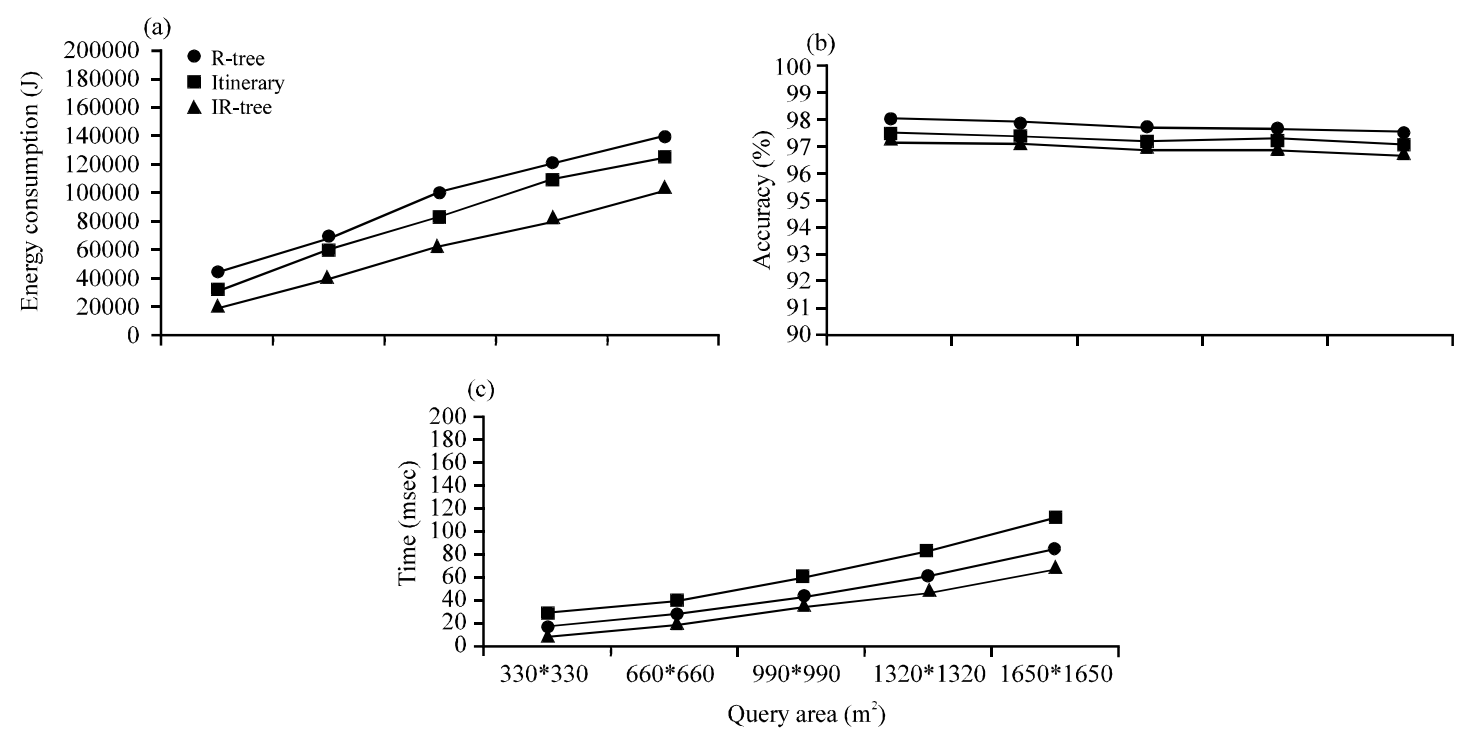

Fig. 19: Evaluation of performance according to size of query area: a) Energy consumption; b) Accuracy of processing results and c) Query processing time

\begin{tabular}{|c|c|c|c|} 
(a) & Pure R-tree & Pure ltinerary & IR-tree \\
\hline $\begin{array}{c}\text { Query } \\
\text { merging }\end{array}$ & None & None & Refelection \\
\hline $\begin{array}{c}\text { Query } \\
\text { parallelism } \\
\text { in query } \\
\text { area }\end{array}$ & $\begin{array}{c}\text { None } \\
\text { procenting }\}\end{array}$ & $\begin{array}{c}\text { None } \\
\text { proquential } \\
\text { processing }\}\end{array}$ & $\begin{array}{c}\text { Parallel } \\
\text { processing }\end{array}$ \\
\hline
\end{tabular}

(b)
\begin{tabular}{|c|c|c|l|}
\hline Division & Pure R-tree & Pure ltinerary & IR-tree \\
\hline Accuracy & Height & Lowness & Middle \\
\hline $\begin{array}{c}\text { Energy } \\
\text { consumption }\end{array}$ & Height & Middle & Low \\
\hline $\begin{array}{c}\text { Processing } \\
\text { time }\end{array}$ & Middle & Slow & Fast \\
\hline
\end{tabular}

Fig. 20: a, b) Results of performance evaluation between R-tree, itinerary and IR-tree

\section{CONCLUSION}

The wireless sensor network is composed of a plurality of sensor nodes (sensor nodes). Sensor nodes are composed of sensing (sensing), processing (processing), storage (storage) and communication (communicating) modules which are characterized by very low energy capacity and energy consumption during data transfer. For this reason, in wireless sensor networks, researches are being carried out to efficiently process various spatio-temporal queries with a sensor having a small amount of energy according to the characteristics of such wireless sensor nodes. In particular, processing of spatio-temporal queries for acquiring necessary data from a large number of wireless sensors attracts attention in important research fields. The spatial operation query processing is a method of processing queries such as temperature, humidity, illuminance, etc., for the entire wireless sensor network or a specific area.

The simplest method of spatial query processing in these wireless sensor networks is the centralized method of collecting the location and sensing information of all the sensor nodes collectively at the server and processing the query of the space on the server. Although, this method has an advantage of being simple there is a disadvantage that the energy efficiency of the sensor node is greatly reduced due to the high wireless communication cost required for accessing all the wireless sensor nodes. In order to complement these centralizedmethods, an in-network based distributed spatial index method has been proposed that performs distributed spatial filtering at sensor nodes to 
reduce the number of wireless communications between sensor nodes. However, since, these distributed spatial index methods are simply applications for sensor networks, existing spatial index methods used in most servers are optimized simultaneously in the in-network environment and the effects of spatial filtering and wireless routing between sensor nodes have no drawbacks.

Therefore, in this study, we solve the problem of such a spatial query processing technique and present IR-tree (Itinerary based R-tree) for more efficient query processing in wireless sensor network. In the creation of an IR-tree in a specific sensor space region, a center node (center node) is first selected according to the wireless sensor node distribution to form an R-tree based on MBR (Minimum Bounding Rectangle). Subsequently, the quality region is constructed with a quadtree to improve accuracy and retrieval speed. The MBR of the R-tree can be overlapped and the MBR of the upper node is a hierarchical tree structure including the MBR of the child node. Index structure during query processing, a specific upper level searches R-trees and uses the Itinerary method from most lower levels.

\section{ACKNOWLEDGEMENT}

This research was supported by the National Research Foundation of Korea (NRF) grant funded by the Korea government (MSIP) (No. 2017R1A2B4011243).

\section{REFERENCES}

Akkaya, K. and M. Younis, 2005. A survey on routing protocols for wireless sensor networks. Ad Hoc Networks, 3: 325-349.

Bash, B.A., J.W. Byers and J. Considine, 2004. Approximately uniform random sampling in sensor networks. Proceeedings of the 1st International Workshop on Data Management for Sensor Networks: In Conjunction with VLDB 2004 (DMSN'04), August 30, 2004, ACM, Toronto, Canada, pp: 32-39.
Culler, D., D. Estrin and M. Srivastava, 2004. Guest editor's introduction: Overview of sensor networks. Computer, 37: 41-49.

Gang, H.G., J.J. Kim and G.J. Han, 2007. Non-uniform network segmentation scheme considering energy efficiency in data centersensor network. J. Korea Spatial Inf. Syst. Soc., 9: 35-50.

Guttman, A., 1984. R-trees: A dynamic index structure for spatial searching. Proceedings of the 1984 ACM SIGMOD International Conference on Management of Data (SIGMOD'84), June 18-21,1984, ACM, Boston, Massachusetts, USA., ISBN:0-89791-128-8, pp: 47-57.

Karp, B. and H.T. Kung, 2000. GPSR: Greedy peimeter stateless routing for wireless networks. Proceedings of the 6th Annual International Conference on Mobile Computing and Networking, August 6-11, 2000, Boston, MA., USA., pp: 243-254.

Kim, J.J., I.S. Shin, K.Y. Lee and K.J. Han, 2011. Efficient processing of aggregate queries in wireless sensor networks. J. Korean Spatial Inf. Soc., 19: 95-106.

Kim, K.T. and H.Y. Youn, 2010. Tree-based clustering protocol for energy efficient wireless sensor networks. KIPS. Trans. Part C., 17: 69-80.

Kim, M.S. and I.S. Jang, 2011. Energy efficient distributed space indexing technique in wireless sensor networks. J. Korean Spatial Inf. Soc., 19: 63-74.

Soo, K.J., P. Chan-Hee, K.J. Keun and K.B. Wook, 2007. Energy-efficient data aggregation using hierarchical filtering in sensor networks. J. Korea Comput. Inf. Soc., 12: 79-88. 